International Journal of Biomedicine I June 2019 - Volume 9, Issue Suppl_1: Abstracts From the Second Russian International Conference "Cryo-electron microscopy 2019: achievements and prospects"

ORAL ABSTRACT PRESENTATIONS

SESSION TITLE: COMPLEX AND EMERGING TECHNIQUES IN STRUCTURAL BIOLOGY

DOI: 10.21103/IJBM.9.Suppl_1.OR21

\title{
Abstract OR-21: 3D Structure of the Natural Tetrameric Form of Human Butyryl- cholinesterase as Revealed by Cryo-EM, MD and SAXS
}

Konstantin M. Boyko', Timur N. Baymukhametov ${ }^{2}$, Yury M. Chesnokov², Michael Hons ${ }^{3}$, Sofya V. Lushchekina $^{4}$, Alexey V. Lipkin ${ }^{1}$, Vladimir O. Popov ${ }^{1,2}$, Michail V. Kovalchuk ${ }^{2}$

${ }^{1}$ Bach Institute of Biochemistry, Research Center of Biotechnology of the Russian Academy of Sciences, Moscow, Russia; ${ }^{2}$ National Research Center «Kurchatov Institute», Moscow, Russia; ${ }^{3}$ EMBL Grenoble,

Grenoble, France; ${ }^{4}$ Emanuel Institute of Biochemical Physics, Russian Academy of Sciences, Moscow, Russian Federation

Background: Human plasma butyrylcholinesterase (hBChE, EC 3.1.1.8) is an endogenous bioscavenger that hydrolyzes numerous medicamentous and poisonous esters and scavenges potent organophosphorus nerve agents. Thus, the hBChE can be used to protect acetylcholinesterase as well as a marker for diagnosis of OP poisoning. It is also considered as a therapeutic target against Alzheimer's disease. Though the X-ray structure of a partially deglycosylated monomer of human hBChE was solved 15 years ago, all attempts to determine the 3D structure of the natural full-length glycosylated tetrameric human hBChE were unsuccessful so far.

Methods: A combination of three complementary structural methods - single particle cryoelectron microscopy, molecular dynamic simulations and small-angle X-ray scattering were implemented to elucidate the overall structural and spatial organization of the natural tetrameric human plasma $\mathrm{hBChE}$.

Results: A $7.6 \AA$ cryo-EM map clearly shows the structural organization of the enzyme: a dimer of dimers with a non-planar monomer arrangement in which the interconnecting super helix complex PRAD-(WAT)4-peptide C-terminal tail is located in the center of the tetramer, nearly perpendicular to its plane and deeply plunged between the four subunits. Time-averaged molecular dynamics trajectories allowed to optimize the geometry of the molecule and to reconstruct structural features obscured in the cryoEM density, e.g glycan chains and glycan inter-dimer contact areas as well as inter-monomer disulfide bridges at the C-terminal tail. Finally, SAXS data confirmed the consistency of the obtained model with the experimental data.

Conclusion: The 3D structure of human tetrameric butyrylcholinesterase, obtained for the first time using a combination of complementary structural methods, revealed unique quaternary organization distinct from the model proposed previously. 
Key Words: butyrylcholinesterase $\bullet$ tetramer $\bullet$ cryo-EM $\bullet$ bioscavenger

Sources of Funding: This work was supported by the Russian Science Foundation projects - \#19-1400164 (in part of the data collection and analysis), \#18-41-06001 (in part of image processing) and \#1714-01097 (in part of the sample preparation) as well as by Russian Foundation for Basic Research project \#19-03-00043 (in part of molecular dynamics simulation).

International Journal of Biomedicine. 2019;9 Suppl 1: S14-15. doi: 10.21103/IJBM.9.Suppl_1.OR21

C2019 International Medical Research and Development Corporation 